\title{
A splicing mutation affecting expression of ataxia-telangiectasia and Rad3-related protein (ATR) results in Seckel syndrome
}

\author{
Mark O’Driscoll ${ }^{1 \star}$, Victor L. Ruiz-Perez ${ }^{2 \star}$, C. Geoffrey Woods ${ }^{3}$, Penny A. Jeggo ${ }^{1}$ \& Judith A. Goodship ${ }^{2}$ \\ ${ }^{*}$ These authors contributed equally to this work.
}

Published online 17 March 2003, doi:10.1038/ng1129

\begin{abstract}
Seckel syndrome (OMIM 210600) is an autosomal recessive dis- below the mean). Our analysis shows that UV-induced ATR actiorder characterized by intrauterine growth retardation, vation can occur in non-replicating cells following processing by dwarfism, microcephaly and mental retardation. Clinically, nucleotide excision repair.

Seckel syndrome shares features in common with disorders Genetic mapping studies identified ATR as a candidate gene for involving impaired DNA-damage responses, such as Nijmegen Seckel syndrome, but its location on the physical map had not breakage syndrome (OMIM 251260) and LIG4 syndrome (OMIM been defined. We used the ATR cDNA sequence to identify a 606593). We previously mapped a locus associated with Seckel $112-\mathrm{kb}$ genomic sequence ${ }^{4}$ that in turn retrieved two linked syndrome to chromosome 3q22.1-q24 in two consanguineous BACs, one of which was located at 147.77 Mb on chromosome 3,
\end{abstract} Pakistani families ${ }^{1}$. Further marker analysis in the families, including a recently born unaffected child with a recombination in the critical region, narrowed the region to an interval of $5 \mathrm{Mbp}$ between markers D3S1316 and D3S1557 (145.29 Mbp and $150.37 \mathrm{Mbp}$ ). The gene encoding ataxia-telangiectasia and Rad3-related protein (ATR) maps to this region ${ }^{2,3}$. A fibroblast cell line derived from an affected individual displays a defective DNA damage response caused by impaired ATR function. We identified a synonymous mutation in affected individuals that alters ATR splicing. The mutation confers a phenotype including marked microcephaly (head circumference 12 s.d. below the mean) and dwarfism (5 s.d.

Fig. 1 F02-98 cells show an impaired response to DNA damage. a, F02-98 cells were impaired in phosphorylation of $\mathrm{H} 2 \mathrm{AX}$ $(\gamma \mathrm{H} 2 \mathrm{AX})$ and $\mathrm{p} 53 \mathrm{Ser} 15$ induced by ultraviolet radiation (UV) but normal in phosphorylation of these substrates after exposure to ionizing radiation (IR). Owing to difficulty in obtaining sufficient material from primary fibroblast cells for western blotting, the phosphorylation of ATR substrates after exposure to DNAdamaging agents was examined by immunofluorescence using phosphospecific antibodies ( $\alpha$-P-Ser15-p53). Phosphorylation was examined after exposure to ionizing radiation (10 Gy) and ultraviolet radiation $\left(5 \mathrm{~J} \mathrm{~m}^{-2}\right) 1 \mathrm{~h}$ after irradiation. Cells held under low-serum conditions for $5 \mathrm{~d}$ before exposure to ultraviolet radiation had an identical response to that of exponentially growing cells (data not shown). b, F02-98 cells were impaired in phosphorylation of hRad17 and Nbs1 induced by ultraviolet radiation (UV). The examination of phosphorylation was carried out as described in a using phosphospecific antibodies ( $\alpha$-PRad17 and $\alpha$-P-Nbs1). Immunofluorescence was also analyzed using antibodies that recognize endogenous Rad17 and Nbs 1 $(\alpha$-Rad 17 and $\alpha$-Nbs 1 ; right panels) verifying that these proteins were expressed efficiently before and after ultraviolet radiation treatment in F02-98 cells. $\boldsymbol{c}, \boldsymbol{d}$, F02-98 cells showed impaired expression of ATR protein. ATR expression was examined by western blotting $(c)$ and by immunofluorescence $(d)$. The residual signal seen by immunofluorescence in F02-98 cells could represent background staining or residual protein.

$a$

1BR.3

F02-98

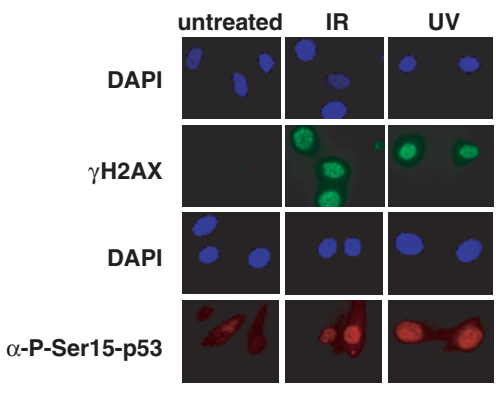

$$
b
$$

1BR.3

F02-98


$c$
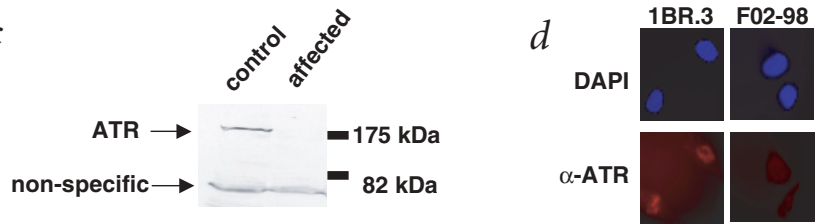

${ }^{1}$ Genome Damage and Stability Centre, University of Sussex, East Sussex, BN1 9RQ, UK. ${ }^{2}$ Institute of Human Genetics, Newcastle University, Newcastleupon-Tyne, UK. ${ }^{3}$ Molecular Medicine Unit, St. James' University Hospital, Leeds, UK. Correspondence should be addressed to P.A.J. (e-mail: p.a.jeggo@sussex.ac.uk). 




$b$

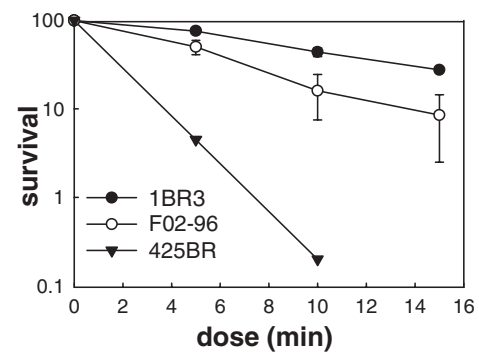

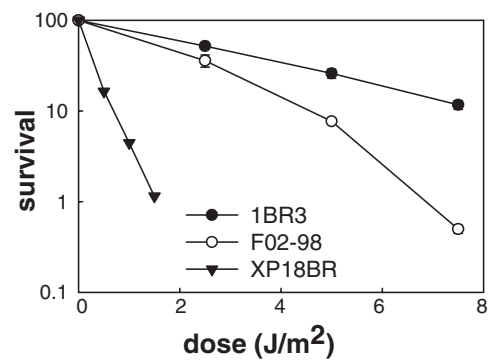

Fig. 2 F02-98 cells are hypersensitive to ultraviolet radiation and mitomycin C. Sensitivity to ionizing radiation (a), mitomycin C (b) and ultraviolet radiation (c) was examined using clonogenic survival assays. The $y$ axis shows the percent survival. The results shown represent the mean \pm s.d. of three experiments for $1 \mathrm{BR} 3$ and F02-98 cells. Where error bars are not visible they lie within the symbols. For comparison, survival representing the mean of two experiments is also shown for 425BR cells, a characterized fanconi anaemia group A cell line, exposed to mitomycin C and for XP-18BR cells, a xeroderma pigmentosum group A cell line, exposed to ultraviolet radiation. Compared with additional control cell lines exposed to these agents, the responses of F02-98 cells to mitomycin C and ultraviolet radiation lie outside the normal range, but the response to ionizing radiation does not (data not shown).

within our refined critical interval for Seckel syndrome. Comparison of the human and mouse maps also indicated that ATR was within this region; transcripts on either side of Atr in the mouse corresponded to transcripts located at $147.7 \mathrm{Mb}$ and 148 $\mathrm{Mb}$ on human chromosome 3.

ATR, like ataxia-telangiectasia mutated (ATM), the protein defective in ataxia-telangiectasia, is a phosphotidylinositol 3kinase-like kinase (for reviews see refs. 5-7). Whereas ATM functions predominantly in response to DNA double-strand breaks induced by ionizing radiation, ATR functions primarily in response to other forms of DNA damage, including stalled replication forks. Current data suggests that after exposure to ultraviolet radiation, ATR phosphorylates similar substrates to those phosphorylated by ATM after ionizing radiation, including $\mathrm{p} 53, \mathrm{Rad} 17, \mathrm{Nbs1}$ and $\mathrm{H} 2 \mathrm{AX}$, a variant form of the histone $\mathrm{H} 2 \mathrm{~A}$ (refs. 8-10).

We investigated ATR function in a fibroblast cell line, F02-98, established from an affected individual. We examined phosphorylation of p53, Rad17, Nbs1 and H2AX induced by ultraviolet radiation by immunofluorescence using phospho-specific antibodies. Formation of phosphorylated H2AX in F02-98 cells was defective after exposure to ultraviolet radiation but normal after exposure to ionizing radiation, whereas control cells phosphorylated $\mathrm{H} 2 \mathrm{AX}$ in response to both ultraviolet and ionizing radiation (Fig. 1a). We also observed impaired phosphorylation of p53, Rad17 and Nbs1 induced by ultraviolet radiation (Fig. 1a,b) and reduced ATR expression (Fig. 1c,d). Finally, F02-98 cells showed normal cellular sensitivity to ionizing radiation, consistent with a role of ATM rather than ATR in response to doublestrand breaks, but moderately enhanced sensitivity to mitomycin $\mathrm{C}$ and ultraviolet radiation (Fig. 2). Together, these data show that F02-98 cells have an impaired response to DNA damage, consistent with impaired ATR function.

Because ATR is essential for development, we anticipated that any mutation in ATR would be hypomorphic and examined the cells for evidence of residual ATR protein and function. Overexposure of the ATR western blot indicated the presence of low
Fig. 3 F02-98 cells show residual ATR expression and protein phosphorylation, and defective phosphorylation can be complemented by ATR CDNA. a, Prolonged exposure of a western blot using antibody against ATR showed a residual band with the mobility of ATR protein. $\boldsymbol{b}$, Dose-response analysis of $\mathrm{H} 2 \mathrm{AX}$ phosphorylation. Although H2AX phosphorylation $(\gamma \mathrm{H} 2 \mathrm{AX})$ was not observed after exposure to up to $20 \mathrm{~J}$ $\mathrm{m}^{-2}$ ultraviolet radiation, a signal was observed in approximately $20 \%$ of F02-98 cells after exposure to $40 \mathrm{~J} \mathrm{~m}^{-2}$ of ultraviolet radiation. The panel shows two positively responding cells. Analysis was carried out $1 \mathrm{~h}$ after exposure to ultraviolet radiation. Residual H2AX phosphorylation can also be observed at prolonged times after exposure to $20 \mathrm{~J} \mathrm{~m}^{-2}$ ultraviolet radiation (data not shown). The residual phosphorylation activity is consistent with the residual ATR protein identified by western blotting but could also be due to the activity of another kinase, such as ATM. c Expression of ATR CDNA restored phosphorylation of p53 induced by ultraviolet radiation. F02-98 cells were transfected with pcDNA-3 expressing full length ATR cDNA (ATR), empty pcDNA-3 or pCDNA-3-ATR-KD (ATR-KD). Cells were exposed to ultraviolet radiation (UV; $5 \mathrm{~J} \mathrm{~m}^{-2}$ ) and examined by immunofluorescence for the presence of phosphorylated p53 (Ser15; $\alpha$-P-p53) $48 \mathrm{~h}$ after transfection. Phosphorylated p53 was only observed after transfections with full length ATR cDNA. No complementation was seen after transfection with the kinase-dead version of ATR (as shown) or with an empty vector (data not shown). Expression of ATR cDNA in control cells did not affect their response to ultraviolet radiation (data not shown). $b$

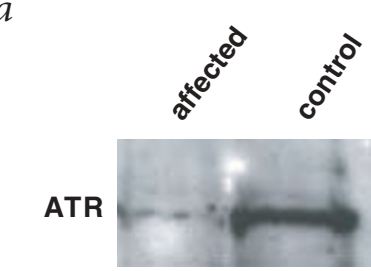

DAPI
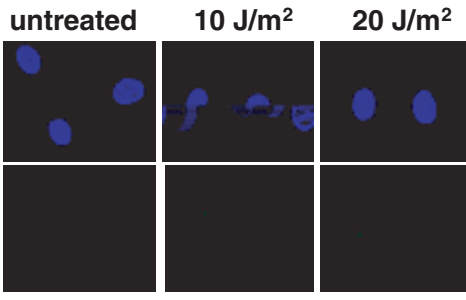

$40 \mathrm{~J} / \mathrm{m}^{2}$

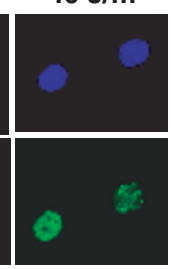

C

F02-98

F02-98 + ATR

F02-98 + ATR-KD
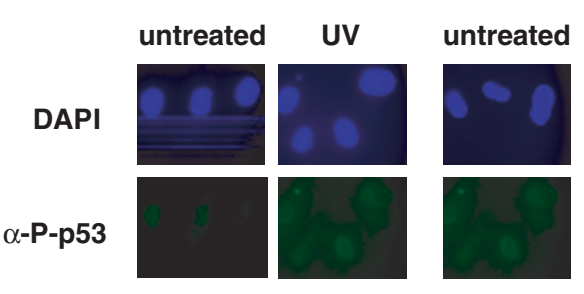

untreated



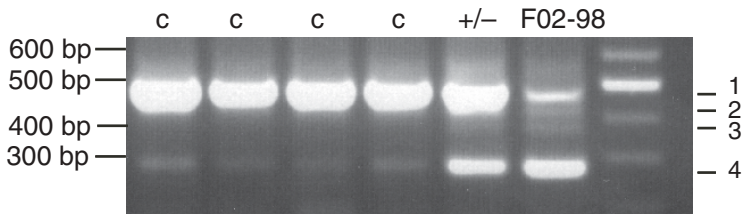

tacataaat taacattagtagaaatct tgtt taatagact taat tt tt t cagCACCACAGGCACAATCACGATGTGTGT TTCTTCTGACTCTGTTTCCAAGAAGAATATTCCTTGAGTGGAGAACAGCAGTTTACAACTGGGCCCTGCAGAGCTCCCAT GAAGTAATCGGGCTAGTTGTGTTAGTGAATTTTTATCTTATTGCAGCAGCAGAATTCTTGTAACAGAGTTCCCAAGAT TCTTATgtatgtactaagattttaatttgaatatatcactctattggaaagatcataattgtctggctatgtatgcaggg ttgtgtttattgttgaatatgtaaagcaaatgttgcttttagttttagaatttaacattttttccttctaggctttgttt taccagtacttttgtcccaaattaagcaagactatttagaccacaaattaggcaagaaagtttagtactttttctttt tttccc CaqAGATAAAGTCAAAGATGATTCTGACATTGTCAAGAAAGAATTTGCTTCTATACTTGGTCAACTTGTCTGTA СTCTTCACGGCATCTTMTATCTCACAAGTTCTTTAACACAACCTTTCTCTCAACACGCACATGTCCACCTCTTCTCTAGC AACTTGAAACCCACTTCTCAACATGAATGTTCATCTTCTCAAAT GAAAAAAAAAATACCTAGTCCAGTAAAACTTGgtgagtgattatgact $b$



control genomic DNA


+ - genomic DNA

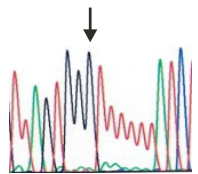

F02-98 genomic DNA

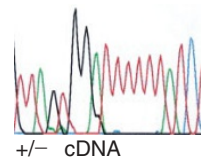

$d$

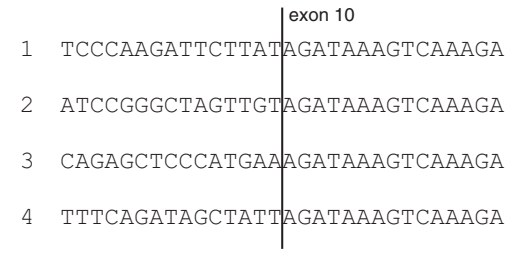

Fig. 4 Identification of a mutation in ATR in individuals with Seckel syndrome. a, Abnormal RT-PCR products using ATR cDNA from F02-98 cells were obtained using primers spanning exon 9. The first four lanes have control cDNA (c) from distinct fibroblast cell lines and the final lane is the size marker. CDNA derived from lymphoblasts from a carrier parent transformed with Epstein-Barr virus is indicated $+/-$. In the control samples, there was a primary product (band 1 ) and a faint band (band 4). The intensity of band 4 is greater in the heterozygote than in the control samples. In F02-98 cells, the intensity of band 4 is greater than that of band 1. Two additional faint bands (bands 2 and 3) were also seen in F02-98 cells. $\boldsymbol{b}$, Sequence analysis of genomic DNA from a control, a heterozygous and an affected individual (F02-98) showing the 2101A $\rightarrow$ G transition (marked by an arrow). In genomic DNA from a heterozygote the A and G peaks were of equal height, as expected. Sequence of band 1 of the CDNA product from the same heterozygote showed that most of the mRNA had the wild-type sequence, though there was a small peak corresponding to the mutant sequence. red, thymine; black, guanine; blue, cytosine; green, adenine. c, Genomic sequence encompassing ATR exons 9 and 10. Intronic sequence is in lower case, exonic sequence in upper case. The normal splice-acceptor and -donor sequences (lower case) and the cryptic splice-donor sequences (upper case) are underlined. The position of the transition is marked by a box. $\boldsymbol{d}$, Abnormal splice products arise from skipping exon 9 or using a cryptic splice site. Bands $1-4$ in a were excised from the gel and sequenced. To the right of the vertical line is exon 10 sequence. Band 1 had normal splicing. Cryptic splice donors were used in bands 2 and 3, the positions of which are shown in c. Band 4 had direct splicing of exon 8 to exon 10.

levels of ATR protein in F02-98 cells (Fig. 3a). A dose-response analysis of phosphorylated H2AX after ultraviolet radiation indicated that there was residual phosphorylation activity after exposure to $40 \mathrm{~J} \mathrm{~m}^{-2}$ (Fig. $3 b$ ). To establish that the phenotype is due to impaired ATR function, we introduced ATR cDNA into F02-98 cells, and we observed correction of the defective phosphorylation of p53 induced by ultraviolet radiation (Fig. $3 c$ ).

We proceeded to sequence the $8.2-\mathrm{kb}$ ATR cDNA by RT-PCR in control and F02-98 fibroblasts using 22 overlapping PCR fragments. The amplification product encompassing exon 9 differed between F02-98 cells, control cells and cells from a parent who carried the mutation (Fig. $4 a$ ). cDNA sequencing indicated that the primary, smaller product in F02-98 cells resulted from loss of exon 9. We then carried out genomic DNA sequencing of exon 9 and the adjacent intronic sequence $(-195$ through to exon 10) to identify the cause of aberrant splicing.
This indicated that the affected individual was homozygous with respect to a single base change, $2101 \mathrm{~A} \rightarrow \mathrm{G}$ (numbering from the first base of mRNA U76308; Fig. 4b). This translationally silent (synonymous) mutation segregated with the disease in both affected families ${ }^{1}$. The remaining ATR cDNA had no other unique changes. We did not find the $2101 \mathrm{~A} \rightarrow \mathrm{G}$ mutation in any of 204 ethnically matched chromosomes.

We also observed two faint intermediately sized bands in F0298 cells (Fig. 4a). Cloning and sequencing showed that these arose by the use of two cryptic splice-donor sites in exon 9 (Fig. $4 c, d$ ). Loss of exon 9 and use of the cryptic splice-donor sites introduces a stop codon in the next exon. A faint band corresponding in size to the product lacking exon 9 was also present in the normal controls, suggesting leaky splicing of this exon. The first evidence confirming that the mutation $2101 \mathrm{~A} \rightarrow \mathrm{G}$ causes aberrant splicing came from sequencing the normal sized cDNA product from a $a$

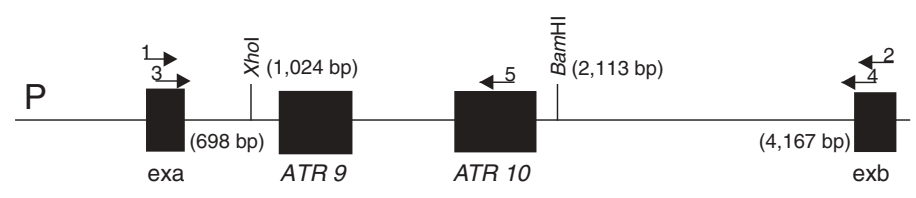

$b$



Fig. 5 In vivo splicing assay shows that the $2101 \mathrm{~A} \rightarrow \mathrm{G}$ transition affects splicing. a, Wild-type and mutated ATR genomic sequences were cloned into the exontrapping vector pSPL3 using the Xhol and BamHI sites. exa and exb, exonic vector sequences; P, promoter; 1-4, primers SD6, SA2, SD2 and SA4; 5, an ATR exon 10-specific oligonucleotide. $\boldsymbol{b}$, Products after amplification of cDNA generated from Cos-7 cells with primers 3 and 5 . The first lane contains the size marker. $C$, negative control using non-transfected cells; $\mathrm{n}$, products from two independent transfections of wild-type construct; $\mathrm{m}$, products from two independent transfections of the mutant construct. The band corresponding to exon 9 inclusion is $477 \mathrm{bp}$, and that corresponding to exon 9 skipping is $284 \mathrm{bp}$. Both products were seen in the lanes corresponding to wild-type sequence $(n)$, but there was no normally spliced product in the lanes corresponding to the mutant sequence ( $m$ ). The same results were observed using primers 3 and 4 (data not shown). 
untreated

$a$

DAPI
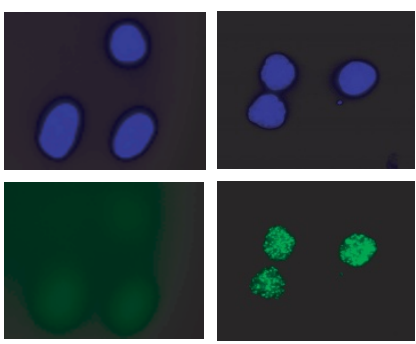

$b$

DAPI
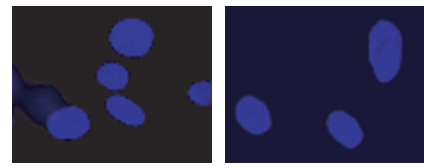

$\mathrm{H} 2 \mathrm{AX}$


c

DAP


$\mathrm{H} 2 \mathrm{AX}$

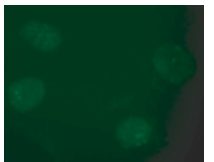

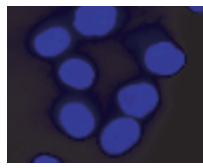
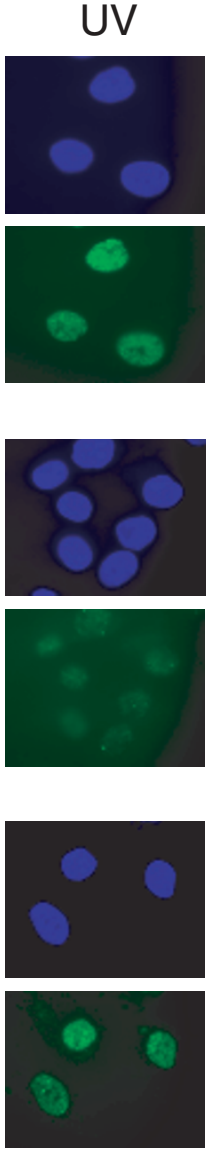

parent carrying the mutation. The mutated allele was underrepresented (Fig. $4 b$ ), indicating that the mutation leads to exon skipping but that some of the RNA is correctly spliced.

To confirm the impact of the mutation $2101 \mathrm{~A} \rightarrow \mathrm{G}$ on splicing efficiency, we constructed wild-type and mutated ATR hybrid minigenes in an exon-trapping vector, transfected these into Cos-7 cells and analyzed the products by RT-PCR and sequencing (Fig. 5). The wild-type minigene generated a primary band reflecting exon 9 inclusion and a minor product lacking exon 9 , whereas the mutated minigene generated only the product missing exon 9 . These data show that correct splicing of exon 9 is inefficient in wildtype DNA and that the $2101 \mathrm{~A} \rightarrow \mathrm{G}$ mutation markedly reduces splicing efficiency. Notably, we observed a low level of correctly spliced transcript from F02-98 cells, indicating that the mutation does not fully abolish correct splicing and consistent with the residual activity and protein observed in F02-98 cells.

Shapiro and Senapathy have devised a system for evaluating splice-site efficiency in which efficient splice sites have a score $>94$ (ref. 11). The ATR exon 9 splice-acceptor site has a polyU tract interrupted by two adenines, which slightly reduces the score for acceptor sequence efficiency from 94 to 90 . The ATR exon 9 splice-donor site has a markedly low score of 76 . The mutational change does not create a new efficient splice site. Splicing, particularly when splice sites are weak, is influenced by exon-splice enhancers (ESEs) and silencers (ESSs). It is possible that the mutation disrupts an ESE or creates an ESS. Analysis of exon 9 using ESEfinder programs did not identify any known ESE sequence encompassing the mutation (refs. 12,13; W. Fairbrother, pers. comm.). The RNA modeling program mfold showed that the mutation could potentially influence the secondary structure of the transcript.
Fig. 6 ATR activation by ultraviolet radiation in non-replicating cells requires processing by nucleotide-excision repair. Control fibroblasts and fibroblasts derived from an individual with xeroderma pigmentosum group A (XP40BR) or an individual with Cockayne syndrome (CS11BR) were exposed to ionizing radiation (IR; $10 \mathrm{~Gy}$ ) or ultraviolet radiation (UV; $10 \mathrm{~J} \mathrm{~m}^{-2}$ ) and examined for $\mathrm{H} 2 \mathrm{AX}$ phosphorylation $(\gamma \mathrm{H} 2 \mathrm{AX})$ after $1 \mathrm{~h}$. a, 1BR3 (control fibroblasts); $\boldsymbol{b}$, XP40BR; c, CS11BR. Phosphorylation of H2AX $(\gamma \mathrm{H} 2 \mathrm{AX})$ was evident in control and CS11BR cells but was completely absent in XP40BR cells as it was in F02-98 cells (shown in Fig. 1). Identical results were obtained with XP4LO and GM01629, other cell lines from individuals with xeroderma pigmentosum group A and Cockayne syndrome, respectively (data not shown).

The DNA replication checkpoint is ATR-dependent, and recent studies have proposed a requirement for replication for the activation of ATR after DNA damage ${ }^{14,15}$. Our data, however, suggest that ATR activation can occur in G1-phase human cells exposed to ultraviolet radiation. We used a cell line defective in ATR to examine whether ATR activation occurs in the absence of replication. We maintained normal cells in G0/G1 phase by serum starvation and irradiated them with $5 \mathrm{~J} \mathrm{~m}^{-2}$ ultraviolet radiation. These non-replicating cells efficiently phosphorylated H2AX (Fig. 1). We did not observe phosphorylation in parallel experiments with F02-98 cells, confirming the ATR dependence of the phosphorylation. To gain insight into the requirement for ATR activation, we examined phosphorylation in cell lines impaired in the processing of lesions induced by ultraviolet radiation. H2AX phosphorylation was not observed in two cell lines derived from individuals affected with xeroderma pigmentosum group A and defective in nucleotide-excision repair but was evident in two cell lines derived from individuals with Cockayne syndrome and defective in transcription-coupled repair (Fig. 6). Taken together, these findings indicate that ATR-dependent signaling, including phosphorylation of $\mathrm{H} 2 \mathrm{AX}$, can be activated in a replication-independent manner after nucleotide-excision repair of photoproducts from ultraviolet radiation.

Our findings identify a founder mutation $(2101 \mathrm{~A} \rightarrow \mathrm{G})$ in ATR in two families affected with Seckel syndrome and an impaired response to DNA damage in a cell line from an affected individual. This synonymous mutation has a profound affect on splicing efficiency, resulting in reduced but residual levels of normal transcript and protein. The severity of this hypomorphic mutation is shown by the marked microcephaly and dwarfism observed in affected individuals and is consistent with the embryonic and somatic lethality seen in the absence of ATR ${ }^{16,17}$. Our findings, therefore, provide the first evidence of a clinical disorder associated with impaired ATR signaling and raise the possibility that mutations in additional components of the ATR signaling pathway could contribute to further instances of Seckel syndrome.

\section{Methods}

Families and genotyping. Family 1 and family 2 are consanguineous Pakistani families that have been described previously ${ }^{1}$. Microsatellite markers D3S3023, D3S1316, D3S3694, D3S1569, D3S1557, D3S1593 and D3S3622 were amplified and analyzed on an ABI377. We used a control panel of 105 unrelated Pakistani individuals. Cells were obtained from family members with informed consent. Experiments were approved by the County Durham and Darlington Local Research Ethics Committee.

Mutation analysis. We used lymphoblastoid cell lines and primary fibroblasts as a source of RNA. We propagated lymphoblasts and fibroblasts in RPMI 1640-Glutamax supplemented with $10 \%$ fetal calf serum or in Chang's medium with $2 \mathrm{mM}$ Glutamine, respectively. We isolated total RNA by the TRIZOL protocol (Invitrogen) and used $4 \mu \mathrm{g}$ to synthesize random primed cDNA (Superscript II, Invitrogen). We designed 22 primer pairs producing overlapping fragments to amplify the ATR coding region (details available on request). We analyzed PCR products by gel electrophoresis and sequenced them on an ABI 377 or a Megabace 500. To 
sequence the splicing variants, we purified products (using Qiagen gel extraction kit), TA-subcloned them into pGEM-TEasy (Promega) and carried out plasmid sequencing. To investigate the splice variant, we amplified genomic DNA and sequenced intron 8 (-195 exon 9$)$ through exon 10 . We examined the site of the mutation by sequencing genomic DNA from all members of family 1 and family 2 and from the unaffected controls.

pSPL3-ATR hybrid-minigenes. We used Pfu to amplify 1,089 bp of ATR genomic DNA from intron 8 (-195 exon 9) to intron $10(+202$ exon 10) from an individual heterozygous with respect to the $2101 \mathrm{~A} \rightarrow \mathrm{G}$ mutation. The resulting PCR products were blunt end-subcloned in pCR Blunt (Invitrogen). After sequencing, we subcloned the normal and $2101 \mathrm{~A} \rightarrow \mathrm{G}$ mutated DNA fragments into the multiple cloning site of the exon-trapping vector pSPL3 (Life Technologies) using XhoI and BamHI.

Transfections and RT-PCR. We transiently transfected Cos-7 and HeLa cells using Lipofectamine 2000 (Invitrogen) and then $24 \mathrm{~h}$ later extracted RNA with TRIZOL and generated cDNA using the pSPL3 (Life Technologies) oligonucleotide SA2. We carried out six PCR cycles with vector primers (SD6, $\mathrm{SA2}$ ) before adding two nested primers (SD2, SA4) or one nested vector primer (SD2) in combination with an ATR exon-10 oligonucleotide and carrying out 30 more amplification cycles (Fig. 5). We confirmed the sequence of the splicing products by subcloning into pGEM-TEasy and sequencing.

Cellular analysis. 1BR3 is a control primary fibroblast cell line and F02-98 is a fibroblast line established from an individual from family 1 affected with Seckel syndrome ${ }^{1}$. We cultured cells in minimal essential medium and analyzed their sensitivity to $\gamma$ irradiation, ultraviolet radiation and mitomycin $C$ as described previously ${ }^{18-20}$. To evaluate mitomycin $C$ survival, we exposed exponentially growing cells to $1 \mu \mathrm{g} \mathrm{ml}^{-1}$ mitomycin C in phosphate-buffered saline A for the times indicated, then washed, trypsinized and plated them for survival. We carried out immunofluorescence analysis using the following phospho-specific antibodies: antibody against phospho-histone H2AX (Ser139; Upstate Biotechnology), antibody against phospho-Ser15-p53 (Cell Signalling Technology; Santa Cruz Biotechnology), antibody against phospho-Rad17 (Ser637; a gift from Steve Elledge), antibody against phosphonibrin (Ser343; Santa Cruz Biotechnology), antibody against Rad17 (H-300; Santa Cruz Biotechnology) and antibody against Nbs1 (Ab-1; Oncogene Research Products). The antibodies against ATR that we used for immunofluorescence and western blotting were AHp386 (Serotec). We used a lymphoblastoid cell line established from the affected individual for ATR western blotting. The control represents a lymphoblastoid cell line from an unaffected individual. To arrest primary fibroblast cell lines in G0, we seeded cells onto coverslips and incubated them in MEM medium (Sigma) containing $0.5 \%$ fetal calf serum for at least $5 \mathrm{~d}$. We complemented cells by transient transfection of primary fibroblasts using Genejuice (Novagen) and $2 \mu \mathrm{g}$ pcDNA3-ATR or pcDNA-3 expressing a kinase-dead version of ATR cDNA (pc-DNA3-ATR-KD; ref. 3). We analyzed cells for phosphorylated H2AX foci induced by ultraviolet radiation $48 \mathrm{~h}$ after transfection.

Accession numbers. Mapping the ATR cDNA identified AQ487882, one end of BAC RCP11-262I7. The opposite end of this BAC, AQ487879, was located at $147.77 \mathrm{Mb}$ on chromosome 3, in our refined Seckel syndrome critical interval.

\section{Acknowledgments}

We thank A. Krainer and W. Fairbrother for discussions and A. Carr for the gift of ATR cDNA. This work was supported by the Medical Research Council, the Human Frontiers Science Programme, the Department of Health, the Primary Immunodeficiency Association, the Leukaemia Research Fund, the Wellcome Trust and Newcastle Healthcare Charity. M.O'D. is supported by the Leukaemia Research Fund.

\section{Competing interests statement}

The authors declare that they have no competing financial interests.

Received 21 January; accepted 20 February 2003.

1. Goodship, J. et al. Autozygosity mapping of a Seckel syndrome locus to chromosome 3q22.1-q24. Am. J. Hum. Genet. 67, 498-503 (2000).

2. Cimprich, K.A., Shin, T.B., Keith, C.T. \& Schreiber, S.L. cDNA cloning and gene mapping of a candidate human cell cycle checkpoint protein. Proc. Natl. Acad. Sci. USA 93, 2850-2855 (1996)

3. Bentley, N.J. et al. The Schizosaccharomyces pombe rad3 checkpoint gene. EMBO J. 15, 6641-6651 (1996).

4. Venter, J.C. et al. The sequence of the human genome. Science 291, 1304-1351 (2001).

5. Zhou, B.B. \& Elledge, S.J. The DNA damage response: putting checkpoints in perspective. Nature 408, 433-439 (2000).

6. Shiloh, Y. ATM and ATR: networking cellular responses to DNA damage. Curr. Opin. Genet. Dev. 11, 71-77 (2001).

7. Durocher, D. \& Jackson, S.P. DNA-PK, ATM and ATR as sensors of DNA damage: variations on a theme? Curr. Opin. Cell Biol. 13, 225-231 (2001).

8. Ward, I.M. \& Chen, J. Histone H2AX is phosphorylated in an ATR-dependent manner in response to replicational stress. J. Biol. Chem. 276, 47759-47762 (2001).

9. Zou, L., Cortez, D. \& Elledge, S.J. Regulation of ATR substrate selection by Rad17dependent loading of Rad9 complexes onto chromatin. Genes Dev. 16, 198-208 (2002).

10. Burma, S., Chen, B.P., Murphy, M., Kurimasa, A. \& Chen, D.J. ATM phosphorylates histone H2AX in response to DNA double-strand breaks. J. Biol. Chem. 276, 42462-42467 (2001).

11. Shapiro, M.B. \& Senapathy, P. RNA splice junctions of different classes of eukaryotes: sequence statistics and functional implications in gene expression. Nucleic Acids Res. 15, 7155-7174 (1987).

12. Liu, H.X., Cartegni, L., Zhang, M.Q. \& Krainer, A.R. A mechanism for exon skipping caused by nonsense or missense mutations in BRCA1 and other genes. Nat. Genet. 27, 55-58 (2001)

13. Fairbrother, W.G., Yeh, R.F., Sharp, P.A. \& Burge, C.B. Predictive identification of exonic splicing enhancers in human genes. Science 297, 1007-1013 (2002).

14. Lupardus, P.J., Byun, T., Yee, M.C., Hekmat-Nejad, M. \& Cimprich, K.A. A requirement for replication in activation of the ATR-dependent DNA damage checkpoint. Genes Dev. 16, 2327-2332 (2002).

15. You, Z., Kong, L. \& Newport, J. The role of single-stranded DNA and polymerase $\alpha$ in establishing the ATR, Hus1 DNA replication checkpoint. J. Biol. Chem. 30, 27088-27093 (2002).

16. Cortez, D., Guntuku, S., Qin, J. \& Elledge, S.J. ATR and ATRIP: partners in checkpoint signaling. Science 294, 1713-1716 (2001).

17. Brown, E.J. \& Baltimore, D. ATR disruption leads to chromosomal fragmentation and early embryonic lethality. Genes Dev. 14, 397-402 (2000).

18. Arlett, C.F., Green, M.H.L., Priestley, A., Harcourt, S.A. \& Mayne, L.V. Comparative human cellular radiosensitivity: I. The effect of SV40 immortalisation on the $\gamma$ irradiation survival of skin derived fibroblasts from normal individuals and from ataxia-telangiectasia patients and heterozygotes. Int. J. Radiat. Biol. 54, 911-928 (1988).

19. Marcou, Y., D'Andrea, A., Jeggo, P.A. \& Plowman, P.N. Normal cellular radiosensitivity in an adult fanconi anaemia patient with marked clinical radiosensitivity. Radiother. Oncol. 60, 75-79 (2001)

20. Arlett, C.F., Harcourt, S.A., Cole, J., Green, M.H.L. \& Anstey, A.V. A comparison of the response of unstimulated and stimulated T-lymphocytes and fibroblasts from normal, xeroderma pigmentosum and trichothiodystrophy donors to the lethal action of UV-C. Mutat. Res. 273, 127-135 (1992). 